\title{
Intravesical Infusion of Budesonide Foam Improves Symptoms in a Bladder Pain Syndrome/Interstitial Cystitis Rat Model
}

\section{Kimio Sugaya ${ }^{*}$, Saori Nishijima1, Katsuhiko Noguchi' ${ }^{1}$, Shiho Okitsu' ${ }^{2}$, Katsumi Kadekawa1, Kennosuke Karube ${ }^{3}$, Hideyuki Yamamoto²}

${ }^{1}$ Southern Knights' Laboratory, Okinawa, Japan

${ }^{2}$ Department of Biochemistry, Graduate School of Medicine, University of the Ryukyus, Okinawa, Japan

${ }^{3}$ Department of Cytopathology, Graduate School of Medicine, University of the Ryukyus, Okinawa, Japan

Email: *sugaya@med.u-ryukyu.ac.jp

How to cite this paper: Sugaya, K., Nishijima, S., Noguchi, K., Okitsu, S., Kadekawa, K., Karube, K. and Yamamoto, H. (2020) Intravesical Infusion of Budesonide Foam Improves Symptoms in a Bladder Pain Syndrome/Interstitial Cystitis Rat Model. Open Journal of Urology, 10, 123-133.

https://doi.org/10.4236/oju.2020.105014

Received: February 22, 2020

Accepted: April 11, 2020

Published: April 14, 2020

Copyright $\odot 2020$ by author(s) and Scientific Research Publishing Inc. This work is licensed under the Creative Commons Attribution International License (CC BY 4.0).

http://creativecommons.org/licenses/by/4.0/

(c) (i) Open Access

\begin{abstract}
Purpose: Since intravesically administered steroid therapy may treat bladder pain syndrome/interstitial cystitis (BPS/IC) with fewer side effects than the current treatment of orally administered steroids, we investigated whether the intravesical infusion of budesonide foam can effectively treat BPS/IC symptoms in a rat model of tranilast-induced BPS/IC. Methods: There were 6 rat treatment groups ( $\mathrm{n}=8$ per group): control + single or daily saline infusion, tranilast + single or daily saline infusion, and tranilast + single or daily budesonide infusion. All groups except for the controls were fed a tranilast supplemented diet for 5 weeks. Budesonide and saline were infused intravesically. After the BPS/IC rat model underwent single infusion treatments, we measured the paw pain threshold using the von Frey test and continuous cystometry was taken. After two weeks of daily intravesical infusions, we measured locomotor activity and serum cortisol levels; harvested bladders underwent histological analysis. Results: Both pain threshold and locomotor activity were significantly lower in the saline-infused groups receiving the tranilast diet, but there were no differences between the budesonide-infused groups and the controls. The interval between bladder contractions was significantly shorter in the tranilast group than the control or tranilast + budesonide infusion groups. The serum cortisol levels did not change. Hematoxylin-Eosin stainings of the bladder showed thickening of the bladder muscle layer and mucosal edema in the tranilast group, while the tranilast + budesonide infusion group showed only mild changes. Conclusion: Intravesical infusion of budesonide effectively treated BPS/IC symptoms in a rat model of BPS/IC.
\end{abstract}




\section{Keywords}

Bladder Pain, Budesonide, Interstitial Cystitis, Rats, Tranilast

\section{Introduction}

Bladder pain syndrome/interstitial cystitis (BPS/IC) is defined as suprapubic pain associated with bladder filling in the absence of urinary tract infections or other diseases and is typically accompanied by increased daytime and nighttime urination frequency, according to both the European Society for the Study of Interstitial Cystitis (ESSIC) and the European Association of Urology (EAU) [1] [2]. The etiology of BPS/IC is not fully understood yet, but there is evidence that indicates that BPS/IC is an autoimmune disease of the bladder because it is known to be associated with other autoimmune disorders such as systemic lupus erythematosus and allergies [3] [4].

When patients used tranilast to treat allergies, keloids, and hypertrophic scars, some of them sometimes developed BPS/IC [5] [6] [7]. Tranilast, N-(3,4-dimethyoxycinnamoyl) anthranilic acid, treats these diseases by suppressing the release of transforming growth factor (TGF)- $\beta 1$ to inhibit collagen synthesis [8] [9]. We previously developed a BPS/IC rat model by administering high doses of tranilast to rats; our model closely resembles human BPS/IC because it has the typical signs and symptoms experienced by BPS/IC patients, such as increased urination frequency and decreased locomotor activity [10] [11]. Our BPS/IC rat model is also characterized by having an extended bladder that leads to inflammation, increased vascular permeability, and the presence of glomerulations in the bladder wall [10].

Although many systemic and intravesical agents have been investigated for treatment of BPS/IC, it remains a refractory disease. Oral administration of steroids is relatively effective for BPS/IC symptoms, but oral steroid therapy is associated with numerous adverse effects [12] [13]. As an alternative to oral administration, steroid enemas are used for the treatment of ulcerative colitis [14] [15]. Rectabul ${ }^{\varpi}$ (Kissei Pharmaceutical, Matsumoto, Japan) is a steroid enema foam containing $2 \mathrm{mg}$ of budesonide per dose [16]. Since the drug is a foam preparation, it is retained in the rectum and sigmoid colon after infusion with minimal leakage [14] [15]. In addition, local administration of a steroid is expected to reduce systemic side effects.

Since intravesically administered steroid therapy may treat BPS/IC with fewer side effects than the current treatment of steroids administered orally, we investigated whether the intravesical infusion of budesonide foam can effectively control BPS/IC symptoms in a BPS/IC rat model induced by tranilast.

\section{Methods}

Animals 
48 female Sprague Dawley rats weighing 200 to $240 \mathrm{~g}$ were used in this study. This study protocol was approved by the President of the University of the Ryukyus based on recommendations from the Institutional Animal Care and Use Committee (A2017182 and A2019002).

\section{Treatment with a single intravesical infusion of budesonide}

In the first experiment, 24 rats were randomly divided into 3 groups: control + single saline infusion group, tranilast + single saline infusion group, and tranilast + single budesonide infusion group ( $\mathrm{n}=8$ for each group). For 5 weeks, all groups except for the controls were fed a powder diet containing $0.4 \%$ tranilast (Kissei Pharmaceutical, Matsumoto, Japan), and the controls were fed a standard powder diet (Clea Japan, Tokyo, Japan). After 5 weeks of the tranilast diets, rats were anesthetized using isoflurane and transurethral catheterizations were performed using a fine polyethylene catheter (PE50, Clay Adams, NJ, USA). Next, the tranilast + single budesonide infusion group received an intravesical infusion of $0.5 \mathrm{~mL}$ of budesonide foam (Rectabul ${ }^{\circledR}$ ) that contains approximately $2 \mu \mathrm{g}$ of budesonide, and both the tranilast + single saline infusion and control + single saline infusion groups received an intravesical infusion of $0.5 \mathrm{~mL}$ of physiological saline [16].

\section{Von Frey testing}

2 hours after the treatment infusions, the von Frey test was used to measure nociceptive paw pain (BIO-EVF5; PanLab, Holliston, MA, USA) in the three groups.

\section{Continuous cystometry}

We performed continuous cystometry on the single infusion-treated rat groups. The rats were anesthetized using urethane that was administered both subcutaneously $(0.8 \mathrm{~g} / \mathrm{kg})$ and intraperitoneally $(0.4 \mathrm{~g} / \mathrm{kg})$. Physiological saline was infused directly $(0.05 \mathrm{ml} / \mathrm{min})$ into the bladder using a PE50 catheter that was inserted transurethrally into the bladder and was connected to the infusion pump. We recorded bladder activity for a minimum of 90 min during cystometry. We also measured the residual volume after final voiding.

\section{Treatment with multiple daily intravesical infusions of budesonide}

The remaining 24 rats were randomly divided into 3 groups: control + daily saline infusion group, tranilast + daily saline infusion group, and tranilast + daily budesonide infusion group ( $\mathrm{n}=8$ for each group). For 5 weeks, all groups except for the controls were fed a powder diet containing $0.4 \%$ tranilast, and the controls were fed a standard powder diet. After 3 weeks of the tranilast diets, all rats were given daily intravesical infusions for 2 weeks; the tranilast + daily budesonide infusion group received $0.5 \mathrm{~mL}$ budesonide foam, and both the tranilast + daily saline infusion and control + daily saline infusion groups received 0.5 $\mathrm{mL}$ physiological saline.

\section{Locomotor activity testing}

After 2 weeks of daily infused treatments, we measured locomotor activity to evaluate pelvic pain using previously described methods using a digital counter and an infrared sensor (NS-ASS01; Neuroscience Inc., Tokyo, Japan) [10]. 
Nighttime locomotor activity was measured from 8:00 P.M. to 8:00 A.M. with the lights off.

At the end of the experiment, rats were anesthetized for the collection of venous blood samples from the inferior vena cava to measure serum cortisol levels (BML Inc., Tokyo, Japan) and the excision of bladders for histological examination.

\section{Histology}

The bladders of the rats were harvested, visually inspected, fixed in $10 \%$ formalin buffer, processed, embedded, sectioned, Hematoxylin-Eosin stained, and analyzed for histological changes.

\section{Statistical Analysis}

Results are reported as the mean \pm standard error of the mean (SEM). Student's unpaired $t$-test was used to determine significance. A $p<0.05$ indicated significance.

\section{Results}

\section{Nociceptive paw pain threshold}

We used the von Frey test to determine that the mean threshold for nociceptive paw pain was $26.5 \pm 2.2 \mathrm{~g}, 33.6 \pm 2.1 \mathrm{~g}$, and $35.3 \pm 2.6 \mathrm{~g}$ for the tranilast + single saline infusion group, tranilast + single budesonide infusion group, and control + single saline infusion group, respectively ( $\mathrm{n}=8$ for each group) (Figure 1). The mean threshold for nociceptive paw pain was significantly lower in the tranilast + single saline infusion group than in the control + single saline infusion group ( $\mathrm{n}=8, p=0.022)$, and no significant differences were observed

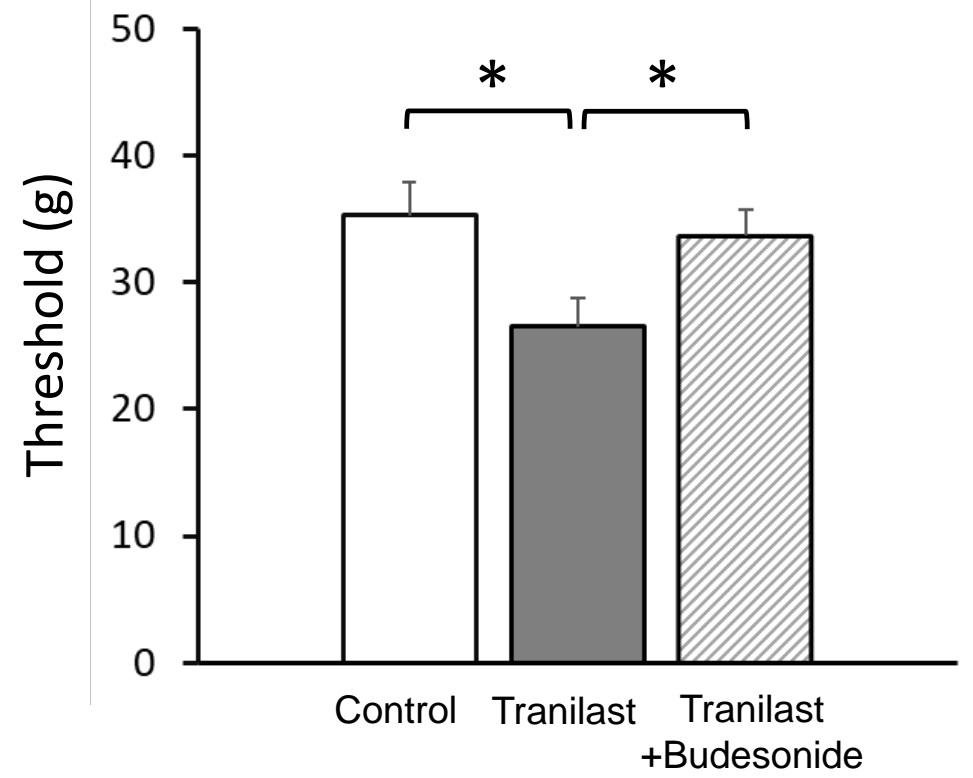

Figure 1. The von Frey test was used to determine the paw pain threshold in the BPS/IC rat model after treatment. Control: control + single saline infusion group, Tranilast: tranilast + single saline infusion group, Tranilast + Budesonide: tranilast + single budesonide infusion group. Mean $\pm \mathrm{SEM}, \mathrm{n}=8$ per group, ${ }^{\star} p<0.05,{ }^{\star *} p<0.01$. 
between the tranilast + single budesonide infusion group and the control + single saline infusion group. Therefore, we demonstrated that the significantly lower nociceptive paw pain threshold induced by tranilast was reversed by a single intravesical infusion of budesonide foam.

\section{Continuous cystometry measurements}

The mean interval between bladder contractions was $15.3 \pm 1$. min, $18.3 \pm 1.7$ min, and $18.9 \pm 1.3 \mathrm{~min}$ for the tranilast + single saline infusion group, tranilast + single budesonide infusion group, and control + single saline infusion group, respectively ( $\mathrm{n}=8$ for each group) (Figure 2 ). The interval between bladder contractions was significantly shorter in the tranilast + single saline infusion group than in the control + single saline infusion group $(p=0.048)$, but no significant differences were observed for the tranilast + single budesonide infusion group. The mean bladder pressure thresholds for inducing bladder contractions were $6.9 \pm 0.5 \mathrm{~cm} \mathrm{H}_{2} \mathrm{O}, 8.3 \pm 0.4 \mathrm{~cm} \mathrm{H}_{2} \mathrm{O}$, and $8.1 \pm 0.3 \mathrm{~cm} \mathrm{H}_{2} \mathrm{O}$ for the tranilast

$\mathrm{AmH}_{\mathrm{Cm}_{2} \mathrm{O}}$
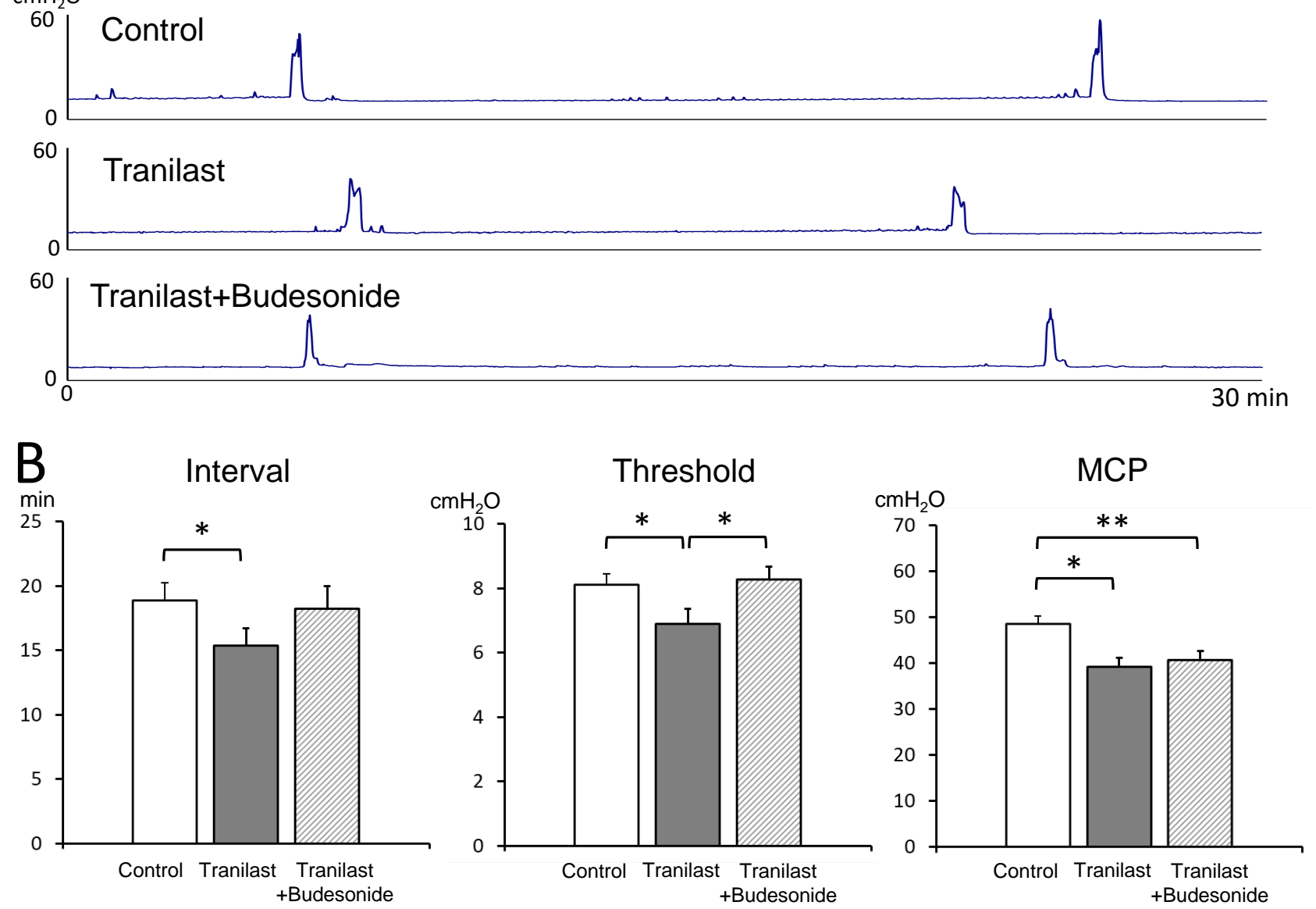

Figure 2. Comparison of continuous cystometry measurements the BPS/IC rat model after treatment. (A) Representative continuous cystometrograms are shown for the 3 treatment groups. (B) The graph shows the interval between bladder contractions (Interval), threshold bladder pressure that induced bladder contractions (Threshold), and the maximum bladder contraction pressure (MCP). Control: control + single saline infusion group, Tranilast: tranilast + single saline infusion group, Tranilast+Budesonide: tranilast + single budesonide infusion group. Mean $\pm \mathrm{SEM}, \mathrm{n}=8$ per group, ${ }^{\star} p<0.05,{ }^{\star *} p<0.01$. 
+ single saline infusion group, tranilast + single budesonide infusion group, and control + single saline infusion group, respectively ( $\mathrm{n}=8$ for each group). The threshold bladder pressure for inducing bladder contractions was significantly lower in the tranilast + single saline infusion group than in the control + single saline infusion group $(p=0.039)$, but no significant differences were observed for the tranilast + single budesonide infusion group. The mean maximum bladder contraction pressure was $39.2 \pm 2.0 \mathrm{~cm} \mathrm{H}_{2} \mathrm{O}, 40.0 \pm 2.0 \mathrm{~cm} \mathrm{H}_{2} \mathrm{O}$, and $48.5 \pm$ $1.7 \mathrm{~cm} \mathrm{H}_{2} \mathrm{O}$ for the tranilast + single saline infusion group, tranilast + single budesonide infusion group, and control + single saline infusion group, respectively ( $n=8$ for each group). The mean maximum bladder contraction pressure was significantly lower in both the tranilast + single saline infusion group and the tranilast + single budesonide infusion group than in the control + single saline infusion group $(p=0.008)$. However, we did not observe any significant differences among the 3 groups for either the baseline bladder pressure that ranged from 3.8 to $7.1 \mathrm{~cm} \mathrm{H}_{2} \mathrm{O}$ or the residual volume that ranged from 0 to $0.2 \mathrm{~mL}$. Thus, the shortened interval between bladder contractions induced by tranilast was reversed with a single intravesical infusion of budesonide foam.

\section{Locomotor activity measurements}

The mean nighttime locomotor activity was $14,510 \pm 568$ movements, 16,863 \pm 1181 movements, and $16,719 \pm 678$ movements for the tranilast + daily saline infusion group, tranilast + daily budesonide infusion group, and control + daily saline infusion group, respectively ( $\mathrm{n}=8$ for each group) (Figure 3 ). The tranilast + daily saline infusion group has significantly less movement than that of the control + daily saline infusion group $(p=0.023)$, but no significant differences were observed for the tranilast + daily budesonide infusion group. Thus, the lower locomotor activity induced by tranilast was reversed by intravesical infusion of budesonide foam.

\section{Serum cortisol measurements}

The mean serum cortisol level was $0.79 \pm 0.04 \mu \mathrm{g} / \mathrm{dL}, 0.75 \pm 0.08 \mu \mathrm{g} / \mathrm{dL}$, and $0.79 \pm 0.05 \mu \mathrm{g} / \mathrm{dL}$ for the tranilast + daily saline infusion group, tranilast + daily budesonide infusion group, and control + daily saline infusion group, respectively ( $\mathrm{n}=8$ for each group). We found no significant differences in the serum cortisol levels among the three groups.

\section{Histological findings of the bladder}

The pathological examination of the excised bladders did not reveal any stones, drug foam, or residue. Thickening of the bladder muscle layer and edema in the bladder wall was observed in the Hematoxylin-Eosin stained sections of the bladder wall from the tranilast + daily saline infusion group, but these changes were less prominent in the tranilast + daily budesonide infusion group and control + daily saline infusion group (Figure 4). We also identified low levels of cellular infiltration into the mucosa in all 3 groups. Thus, the observed histological bladder wall changes induced by tranilast slightly improved after the treatment with budesonide foam. 


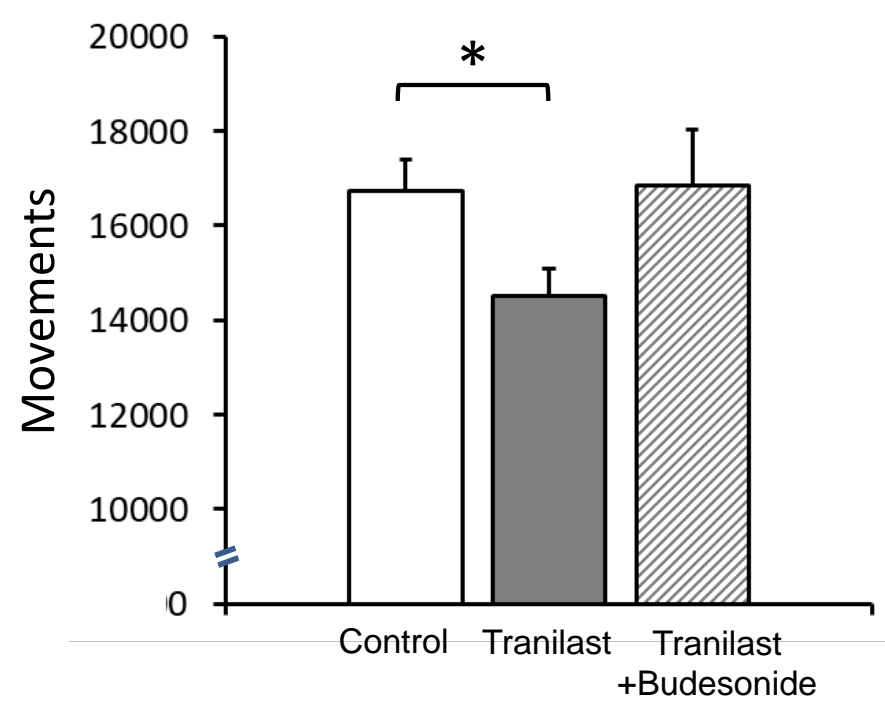

Figure 3. Comparison of nighttime locomotor activity in the BPS/IC rat model after treatment. Control: control + daily saline infusion group, Tranilast: tranilast + daily saline infusion group, Tranilast + Budesonide: tranilast + daily budesonide infusion group. Mean $\pm \mathrm{SEM}, \mathrm{n}=8$ per group, ${ }^{\star} p<0.05$.
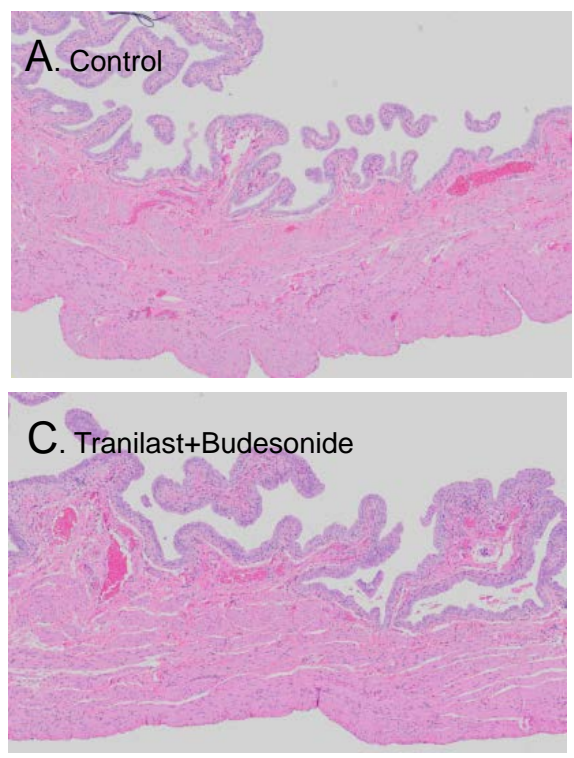

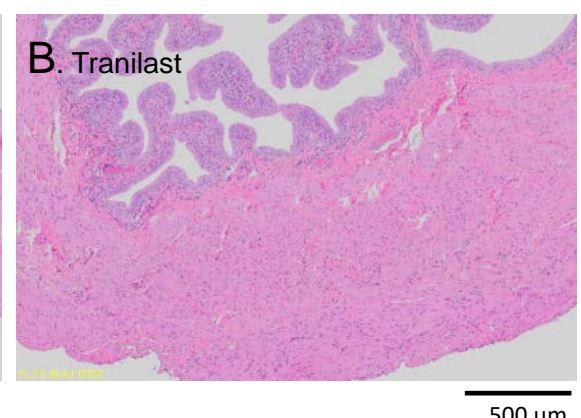

$500 \mu \mathrm{m}$

Figure 4. Histological examination of harvested bladders from BPS/IC rat model after treatment. Representative Hematoxylin-Eosin stainings of the bladder wall are shown for the (A) control + daily saline infusion group, (B) tranilast + daily saline infusion group that has thickening of the bladder muscle layer and mucosal edema, and (C) tranilast + daily budesonide infusion group that has only mild histological changes. Scale bar $=500$ $\mu \mathrm{m}$ for (A), (B), and (C).

\section{Discussion}

In this study, we validated our previously established rat model of BPS/IC by measuring locomotor activity and urinary frequency [10] [11]. We found that rats in the tranilast + daily saline infusion group, who were treated with high doses of tranilast, had significantly less locomotor activity than the control rats. 
Since reduced locomotor activity is an indicator of pelvic pain, this means that our BPS/IC rat model produces pelvic pain symptoms similar to the human version of BPS/IC. Furthermore, continuous cystometry performed on our BPS/IC rat model showed that the interval between bladder contractions was significantly shorter than that of the control group, indicating that our BPS/IC rat model has increased urinary frequency, which is another common symptom of BPS/IC. Therefore, we used our BPS/IC rat model to investigate whether the intravesical infusion of budesonide foam can effectively treat BPS/IC symptoms.

This study showed a lower pain threshold in the nociceptive paw of BPS/IC rats. Intravesical infusion of budesonide foam reversed the lower nociceptive paw pain threshold, reduced locomotor activity, and the shorter interval between bladder contractions that were all induced by the tranilast supplemented diet. Moreover, the histological changes in the bladder wall improved slightly, and no drug residue was observed in the bladders. However, serum cortisol levels were not affected by the intravesical infusion of budesonide foam. Thus, the intravesical infusion of budesonide foam ameliorated the tranilast-induced BPS/IC symptoms in our rat model without increasing serum cortisol levels or causing any adverse influences on the bladder.

In previous studies, we found that the BPS/IC rats had shorter intervals between bladder contractions and thinner bladder walls than control rats, but there were no changes to either the threshold bladder pressure or the maximum bladder contraction pressure [10] [11]. In this study, however, the BPS/IC rats had lower threshold bladder pressure, lower maximum bladder contraction pressure, and thicker bladder walls. These differences from previous studies may have been due to the influence of intravesical catheterization and saline infusion. Since our previous study identified slight inflammatory changes and an increase of bladder wall vascular permeability in the BPS/IC rats fed a tranilast supplemented diet [10], the bladders of these rats might be susceptible to the influence of catheterization and saline infusion. Therefore, it might be possible that intravesical catheterization and saline infusion under tranilast administration caused inflammation of the lower urinary tract, affected intravesical pressure, and thickened the bladder wall. Intravesical infusion of budesonide foam suppressed the bladder wall changes in the BPS/IC rats.

Even though orally-administration steroids can effectively treat BPS/IC, patients experience problematic side effects [12] [13]. Therefore, administering dimethyl sulfoxide (DMSO) [17] [18], heparin [19], hyaluronic acid [20], and bupivacaine with heparin and triamcinolone [18] by intravesical infusions for the treatment of BPS/IC has been investigated both experimentally and clinically, and were reported to be relatively effective with fewer side effects than oral steroids. Another effective BPS/IC treatment involving the endoscopic submucosal bladder injections of either botulinum toxin [21] or the steroid triamcinolone [22] [23] was reported. No major adverse events have been reported for any drugs that were administered by either intravesical infusions or endoscopic injections. However, multiple intravesical infusions and endoscopic submucosal 
injections must be performed, which requires patients to visit clinics for each treatment. On the other hand, enemas can be done at home for the treatment of ulcerative colitis. In the urology field, patients with underactive bladders and large residual urine volumes perform intermittent self-catheterizations. Therefore, it is feasible for BPS/IC patients with severe bladder pain to perform intravesical infusions of steroids by self-catheterization at home.

There were several limitations in our study. The number of animals in each group was small. No data was acquired on possible urinary tract infections that may have occurred, and no data was acquired for possible injuries caused by daily catheterizations. For continuous cystometry, the single transurethral catheterization for the infusion treatment was relatively easy. However, the 2 weeks of daily transurethral catheterizations injured the urethra and caused bleeding that led to difficulties in performing the additional catheterization for cystometry. Therefore, we performed continuous cystometry after only 1 intravesical infusion of either physiological saline or budesonide foam to avoid injuring the urethra while inserting the transurethral catheter. In the future, we plan to investigate the long-term effects of bladder inflammation associated with intravesical steroid infusions performed by self-catheterization in patients with BPS/IC.

\section{Conclusion}

We demonstrated that intravesical infusions of steroid treatment using budesonide foam effectively reduced bladder pain, increased the interval between bladder contractions, and improved histopathological changes in the bladder wall of a BPS/IC rat model. Future clinical investigations on the impact of intravesical steroid treatments by self-catheterization in BPS/IC patients are planned.

\section{Acknowledgements}

We thank Dr. H. Ichise from the University of the Ryukyus, Institute for Animal Experiments, Department of Medicine, for cooperation with this study.

\section{Conflicts of Interest}

The authors declare no conflicts of interest regarding the publication of this paper.

\section{References}

[1] van de Merwe, J.P., Nordling, J., Bouchelouche, P., Bouchelouche, K., Cervigni, M., Daha, L.K., Elneil, S., Fall, M., Hohlbrugger, G., Irwin, P., Mortensen, S., van Ophoven, A., Osborne, J.L., Peeker, R., Richter, B., Riedl, C., Sairanen, J., Tinzl, M. and Wyndaele, J.-J. (2008) Diagnostic Criteria, Classification, and Nomenclature for Painful Bladder Syndrome/Interstitial Cystitis: An ESSIC Proposal. European Urology, 53, 60-67. https://doi.org/10.1016/j.eururo.2007.09.019

[2] Fall, M., Baranowski, A.P., Elneil, S., Engeler, D., Hughes, J., Messelink, E.J., Oberpenning, F., de C Williams, A.C. and European Association of Urology (2010) EAU 
Guidelines on Chronic Pelvic Pain. European Urology, 57, 35-48. https://doi.org/10.1016/j.eururo.2009.08.020

[3] Ochs, R.L., Stein, T.W., Peebles, C.L., Gittes, R.F. and Tan, E.M. (1994) Autoantibodies in Interstitial Cystitis. The Journal of Urology, 151, 587-592. https://doi.org/10.1016/S0022-5347(17)35023-1

[4] Sugaya, K., Nishijima, S., Yamada, T., Miyazato, M., Hatano, T. and Ogawa, Y. (2002) Molecular Analysis of Adrenergic Receptor Genes and Interleukin-4/Interleukin-4 Receptor Genes in Patients with Interstitial Cystitis. The Journal of Urology, 168, 2668-2671. https://doi.org/10.1016/S0022-5347(05)64241-3

[5] Nishida, T., Kusakai, Y. and Ogoshi, R. (1985) Four Cases of Cystitis Induced by the Anti-Allergic Drug Tranilast. Hinyokika Kiyo, 31, 1813-1817.

[6] Okada, H., Minayoshi, K. and Goto, A. (1992) Two Cases of Eosinophilic Cystitis Induced by Tranilast. The Journal of Urology, 147, 1366-1368. https://doi.org/10.1016/S0022-5347(17)37569-9

[7] Sakai, N., Yamada, T. and Murayama, T. (1998) Eosinophilic Cystitis Induced by Tranilast: A Case Report. Hinyokika Kiyo, 44, 45-47.

[8] Suzawa, H., Kikuchi, S., Arai, N. and Koda, A. (1992) The Mechanism Involved in the Inhibitory Action of Tranilast on Collagen Biosynthesis of Keloid Fibroblasts. The Japanese Journal of Pharmacology, 60, 91-96. https://doi.org/10.1254/jjp.60.91

[9] Yamamoto, M., Yamauchi, T., Okano, K., Takahashi, M., Watabe, S. and Yamamoto, Y. (2009) Tranilast, an Anti-Allergic Drug, Down-Regulates the Growth of Cultured Neurofibroma Cells Derived from Neurofibromatosis Type 1. The Tohoku Journal of Experimental Medicine, 217, 193-201.

https://doi.org/10.1620/tjem.217.193

[10] Nishijima, S., Sugaya, K., Kadekawa, K., Ashitomi, K., Ueda, T. and Yamamoto, H. (2013) High-Dose Tranilast Administration to Rats Creates Interstitial Cystitis-Like Symptoms with Increased Vascular Permeability. Life Sciences, 93, 897-903. https://doi.org/10.1016/j.lfs.2013.10.010

[11] Sugaya, K., Nishijima, S., Kadekawa, K., Ashitomi, K., Ueda, T. and Yamamoto, H. (2017) Naftopidil Improves Symptoms in a Rat Model of Tranilast-Induced Interstitial Cystitis. Lower Urinary Tract Symptoms, 9, 107-110.

https://doi.org/10.1111/luts.12113

[12] Hosseini, A., Ehrén, I. and Wiklund, N.P. (2004) Nitric Oxide as an Objective Marker for Evaluation of Treatment Response in Patients with Classic Interstitial Cystitis. The Journal of Urology, 172, 2261-2265. https://doi.org/10.1097/01.ju.0000144761.69398.be

[13] Soucy, F. and Grégoire, M. (2005) Efficacy of Prednisone for Severe Refractory U1cerative Interstitial Cystitis. The Journal of Urology, 173, 841-843. https://doi.org/10.1097/01.ju.0000153612.14639.19

[14] Christophi, G.P., Rengarajan, A. and Ciorba, M.A. (2016) Rectal Budesonide and Mesalamine Formulations in Active Ulcerative Proctosigmoiditis: Efficacy, Tolerance, and Treatment Approach. Clinical and Experimental Gastroenterology, 9, 125-130. https://doi.org/10.2147/CEG.S80237

[15] Naganuma, M., Aoyama, N., Suzuki, Y., Nishino, H., Kobayashi, K., Hirai, F., Watanabe, K. and Hibi, T. (2016) Twice-Daily Budesonide 2-mg Foam Induces Complete Mucosal Healing in Patients with Distal Ulcerative Colitis. Journal of Crohn's and Colitis, 10, 828-836. https://doi.org/10.1093/ecco-jcc/jjv208

[16] KISSEI. https://www.kissei.co.jp/e_contents/news/2017/20171206-814.html 
[17] Perez-Marrero, R., Emerson, L.E. and Feltis, J.T. (1988) A Controlled Study of Dimethyl Sulfoxide in Interstitial Cystitis. The Journal of Urology, 140, 36-39. https://doi.org/10.1016/S0022-5347(17)41478-9

[18] Iyer, S., Lotsof, E., Zhou, Y., Tran, A., Botros, C., Sand, P., Goldberg, R., Tomezsko, J., Gafni-Kane, A. and Botros, S. (2017) Which Bladder Instillations Are More Effective? DMSO vs. Bupivacaine/Heparin/Triamcinolone: A Retrospective Study. International Urogynecology Journal, 28, 1335-1340.

https://doi.org/10.1007/s00192-017-3266-y

[19] Kuo, H.C. (2001) Urodynamic Results of Intravesical Heparin Therapy for Women with Frequency Urgency Syndrome and Interstitial Cystitis. Journal of the Formosan Medical Association, 100, 309-314.

[20] Leppilahti, M., Hellström, P. and Tammela, T.L. (2002) Effect of Diagnostic Hydrodistension and Four Intravesical Hyaluronic Acid Instillations on Bladder ICAM-1 Intensity and Association of ICAM-1 Intensity with Clinical Response in Patients with Interstitial Cystitis. Urology, 60, 46-51.

https://doi.org/10.1016/S0090-4295(02)01613-8

[21] Smith, C.P., Radziszewski, P., Borkowski, A., Somogyi, G.T., Boone, T.B. and Chancellor, M.B. (2004) Botulinum Toxin a Has Antinociceptive Effects in Treating Interstitial Cystitis. Urology, 64, 871-875.

https://doi.org/10.1016/j.urology.2004.06.073

[22] Cox, M., Klutke, J.J. and Klutke, C.G. (2009) Assessment of Patient Outcomes Following Submucosal Injection of Triamcinolone for Treatment of Hunner's Ulcer Subtype Interstitial Cystitis. The Canadian Journal of Urology, 16, 4536-4540.

[23] Funaro, M.G., King, A.N., Stern, J.N.H., Moldwin, R.M. and Bahlani, S. (2018) Endoscopic Injection of Low Dose Triamcinolone: A Simple, Minimally Invasive, and Effective Therapy for Interstitial Cystitis with Hunner Lesions. Urology, 118, 25-29. https://doi.org/10.1016/j.urology.2018.03.037 